\title{
Oral Health and General Health Connections - An Emerging Challenge
}

\section{Koirala S}

Health is the fundamental component for quality of life. Achieving and maintaining health is an ongoing process, shaped by both the evolution of health care knowledge and practices, as well as personal strategies and organized interventions for staying healthy. In developing countries like Nepal, people do not keep oral health maintenance in their priority. However, oral diseases are one of the most common chronic diseases, and are important public health problems because of their prevalence, their impact on individuals and society, and the expense of their treatment. In some countries, oral diseases are the fourth most expensive diseases to treat. Treating caries, estimated at US\$3, 513 per 1000 children, would exceed the total health budget for children of most lowincome countries. ${ }^{1}$ Oral health affects people physically and psychologically and it influences their feelings of social well-being. ${ }^{2}$ The evidence on oral health-general health relationships is growing. In this issue Sharma et al have shown the association of serum LDL cholesterol level with periodontitis. They have found that serum LDL was higher in patients of periodontitis, meaning that there may be higher incidence of coronary artery disease and cerebrovascular disease. ${ }^{3}$ Recent studies have reported associations between oral infections and various systemic diseases - primarily periodontal infections and diabetes, heart disease and stroke, adverse pregnancy outcome, and osteoporosis.

\section{The periodontal disease - diabetic connection:}

The available scientific evidence is particularly strong for a direct relationship between diabetes and periodontal disease. There is a growing acceptance that diabetes is associated with increased occurrence and progression of periodontitis so much that periodontitis has been called "sixth complication of diabetes". ${ }^{4}$ Several studies supported the plausibility that periodontal infections contribute to problem with glycemic control, thus compromising the health of diabetic patients. It has been reported that chronic release of tumor necrosis factor alpha and other cytokines such as those associated with periodontitis interferes with the action of insulin and leads to metabolic alteration. ${ }^{5} \mathrm{~A}$ review article on the interrelationship between periodontitis and diabetes in this issue has explained the association between oral health and systemic disease. ${ }^{6}$

The oral infection, heart diseases and stroke connection:

Recent studies point to an increased risk of heart diseases and stroke in people with periodontal infections. Wu et $\mathrm{al}^{7}$ found periodontal diseases as one of the potential factors for coronary heart disease and stroke based on an analysis of the first National Health and Nutrition Examination Survey and its 21-year follow-up. In this analysis, periodontitis was found to be a significant risk factor for cerebrovascular disease, in particular non-hemorrhagic stroke. Further studies are needed to determine whether periodontal disease alone or in the presence of other oral infections is an independent risk factor for cardiovascular or cerebrovascular disease.

The periodontal disease and adverse pregnancy outcome:

Some studies have found that mothers of preterm, low birth weight infants tend to have more severe periodontal infection than mothers of normal birth weight babies. It is postulated that the periodontal bacteria produces toxins which with other products produced in the mother cross the placenta and harm the fetus. In addition, the response of the maternal immune system to the infection elicits the continued release of inflammatory mediators, growth factors, and other cytokines, which may directly or indirectly, interferes with fetal growth and delivery.

For correspondence

Dr. Sushil Koirala

Vedic Institute of Smile Aesthetics (VISA)

Tel no: 9771-1-4242654

E-mail: skoirala@vedicsmile.com 
Osteoporosis and oral bone Loss:

Osteoporosis, a degenerative bone disease characterized by the loss of bone mineral and associated structural changes, has long been suspected as a risk factor for oral bone loss. In addition, measures of oral bone loss have been proposed as potential screening tests for osteoporosis. ${ }^{8}$

\section{Systemic disease and oral manifestation:}

The mouth can present early warning signs and symptoms in the diagnosis of systemic illnesses and can be predictive of their progression. Oral tissues may also reflect immune deficiency. For example, nearly all HIV- infected individuals develop oral lesions at some time during illness. ${ }^{9}$ Similarly, the appearances of soft or hard tissue pigmentation are associated with a number of diseases like malignant melanoma, Kaposi's sarcoma, Addison's diseases. And treatment procedures like chemotherapy, radiation therapy and variety of pharmaceuticals used to treat systemic diseases can cause oral complications, ranging from xerostomic effects to alteration in the oral hard and soft tissues surface structures.

\section{Saliva based diagnostics:}

The diagnostic value of salivary secretions to detect systemic diseases has long been recognized, ${ }^{10}$ and oral fluids and tissues (buccal cells) are increasingly being used to diagnose a wide range of conditions. Saliva has the potential of replacing blood, the current standard for testing many diseases and conditions.

The evidence on oral health-general health relationships is particularly important in the effort to strengthen integrated oral health promotion and disease prevention around the globe. The recognition of well-known and established signs and symptoms of oral diseases may assist in early diagnosis and prompt treatment of some systemic diseases and disorders. All health professionals and the public should be aware of these signs and symptoms. The associations between oral infections and diabetes, heart disease and stroke, osteoporosis, and adverse pregnancy outcomes warrant a comprehensive and targeted research effort. If any of these associations prove to be causal, major changes in health care delivery and in the training of medical and dental health professionals will be needed.

\section{REFERENCES}

1. Yee R, Sheiham A. The burden of restorative dental treatment for children in Third World countries. Int Dent J. 2002;52:7-10

2. Locker D. Concepts of oral health, disease and the quality of life. In: Slade GD, editor.Measuring oral health and quality of life. Chapel Hill: University of North Carolina, Dental Ecology; 1997, pp. 11-23

3. Sharma S, Lamsal M, Sharma SK, Niraula SR, Koirala B. Association of serum LDL cholesterol level with periodontitis among patients visiting a tertiary-care hospital. JNMA 2011;51(3)104-8.

4. Löe H. Periodontal disease - the sixth complication of diabetes mellitus. Diabetes Care 1993 Jan;16(1):329-34.

5. Pradhan S, Goel K. Interrelationship between Diabetes and Periodontitis: A Review. JNMA 2011;51(3)147-155.

6. Hotamisligil GS, Shargill NS, Spiegelman BM. Adipose expression of tumor necrosis factor-alpha: direct role in obesity-linked insulin resistance. Science 1993 Jan;259(5091):87-91

7. Wu T, Trevisan M, Genco RJ, Dorn JP, Falkner KL, Sempos CT. Periodontal disease and risk of cerebrovascular disease: a prospective study of a representative sample of U.S. adults. Am J Epidemiol 1999;149:(11)290, Suppl S Jun 1.

8. Jeffcoat MK. Osteoporosis: a possible modifying factor in oral bone loss. Ann Periodontol 1998;3:312-21.

9. Greenberg MS. HIV-associated lesions. DermatolClin 1996 Apr;14(2):319-26.

10. Mandel ID. The diagnostic uses of saliva. J Oral Pathol Med 1990;19:119-25. 